Old Dominion University

ODU Digital Commons

Physics Faculty Publications

Physics

$11-2005$

\title{
Alignment Dynamics of Slow Light Diffusion in Ultracold Atomic ${ }^{85} \mathrm{Rb}$
}

S. Balik

Old Dominion University, sbalik@odu.edu

R. G. Olave

Old Dominion University

C. I. Sukenik

Old Dominion University, csukenik@odu.edu

M. D. Havey

Old Dominion University, mhavey@odu.edu

V. M. Datsyuk

See next page for additional authors

Follow this and additional works at: https://digitalcommons.odu.edu/physics_fac_pubs

Part of the Atomic, Molecular and Optical Physics Commons

\section{Original Publication Citation}

Balik, S., Olave, R.G., Sukenik, C.I., Havey, M.D., Datsyuk, V.M., Sokolov, I.M., \& Kupriyanov, D.V. (2005).

Alignment dynamics of slow light diffusion in ultracold atomic ${ }^{85} \mathrm{Rb}$. Physical Review A, 72(5), 51402. doi: 10.1103/PhysRevA.72.051402

This Article is brought to you for free and open access by the Physics at ODU Digital Commons. It has been accepted for inclusion in Physics Faculty Publications by an authorized administrator of ODU Digital Commons. For more information, please contact digitalcommons@odu.edu. 
Authors

S. Balik, R. G. Olave, C. I. Sukenik, M. D. Havey, V. M. Datsyuk, I. M. Sokolov, and D. V. Kupriyanov 


\title{
Alignment dynamics of slow light diffusion in ultracold atomic ${ }^{85} \mathbf{R b}$
}

\author{
S. Balik, R. G. Olave, C. I. Sukenik, and M. D. Havey \\ Department of Physics, Old Dominion University, Norfolk, Virginia 23529, USA \\ V. M. Datsyuk, I. M. Sokolov, and D. V. Kupriyanov \\ Department of Theoretical Physics, State Polytechnic University, 195251 St. Petersburg, Russia
}

(Received 7 October 2005; published 3 November 2005)

\begin{abstract}
A combined experimental and theoretical investigation of time- and alignment-dependent propagation of light in an ultracold atomic gas of atomic ${ }^{85} \mathrm{Rb}$ is reported. Coherences among the scattering amplitudes for light scattering off excited hyperfine levels produce strong variations of the light polarization in the vicinity of atomic resonance. Measurements are in excellent agreement with Monte Carlo simulations of the multiple scattering process.
\end{abstract}

DOI: 10.1103/PhysRevA.72.051402

PACS number(s): 32.80.Pj, 34.80.Qb, 42.50.Gy, 42.50.Nn

Disordered systems have been considered too complex for research into fundamental properties of physical systems. However, technical advances in creation and manipulation of coherence in mesoscopic samples, such as quantum degenerate gases, have made the influence of disorder in atomic and in condensed phases of considerable interest. The essential role disorder can play in phase transitions was pointed out by Anderson [1] in consideration of localization of electrons by disorder. More recent research has focused on general localization phenomena, including localization of matter waves by random or quasirandom optical lattices [2,3]. In this case, the resulting Anderson and Bose glass phases represent a phase transition in the transport properties of the matter waves by optical disorder.

Another research focus area has been localization of electromagnetic waves in strongly scattering and disordered condensed or atomic media [4-7]. Previous studies have considered only massive particles and, in comparison, light localization seems a fundamentally different phenomenon. Nevertheless, there have been two intriguing reports of light localization in condensed samples consisting of classical scatterers $[6,7]$. With characteristicly strong and narrow scattering resonances and well-known interactions with light and external static fields, ultracold atomic gases are being considered as possible systems in which to study light localization. To attain localization, it is generally believed that the Ioffe-Regel condition, $k l \leqslant 1$, must be satisfied [4]. Here $k$ is the local wave vector of the light, and $l$ is the scattering mean free path. For near resonance scattering in an atomic gas, this implies a $\lambda^{-3}$ density scaling, giving a required density larger than $10^{13}$ atoms $/ \mathrm{cm}^{3}$. Physically this means that the light scattering is in the near-field regime. Among other techniques, recent developments in all-optical approaches for forming and manipulating ultracold gases have achieved densities in this range [8]. Light localization studies typically distinguish two limiting cases, one being the strong localization regime where the Ioffe-Regel condition is satisfied. In the weak localization, lower density limit, $k l \gg 1$. Then, for nonquantum degenerate atomic gases, scattering may be thought of as a sequence of scattering and propagation events. However, for ultracold gases in the weak localization regime, quantum interference plays an important role in light transport. Early experiments on interference effects in multiple light scattering in ultracold atomic gases were the measurements, in ultracold atomic ${ }^{85} \mathrm{Rb}$, of coherent backscattering by Labeyrie et al. [9]. In coherent backscattering an interferometric enhancement of the intensity of scattered light is measured in a narrow cone in the nearly backwards direction. The enhancement comes about because reciprocal scattering paths within the medium have phase relations that survive configuration averaging. The experiment was important because it demonstrated the breakdown of a classical description of light transport in an atomic vapor. It also stimulated studies demonstrating interference phenomena associated with magnetic [10], nonlinear optical processes [11], and hyperfine interferences [12].

Although an important goal is to achieve strong light localization in an ultracold atomic gas, all multiple scattering experiments to date have been done in the weak localization limit. The experiments have clarified a number of different features of multiple light scattering in ultracold atomic gases. For example, recent experiments $[13,14]$ reported on the time-evolution of light scattered from optically thick samples of ultracold alkali-metal atoms. In the experiments of Ref. [13] in ${ }^{85} \mathrm{Rb}$, attention was focused on the delay time associated with the process, which consists of a transport and a dwell time $[5,15]$. The combination was shown, in a range on the order of the natural width of the transition, to be independent of detuning. This important result demonstrated the essential roles that scattering and transport processes play in the time scale for light transport. The experiment reported a small diffusive energy velocity $\sim 10^{-5} c$, where $c$ is the vacuum speed of light. Note that this is not the more familiar slow-light behavior observable in the coherent beam due to electromagnetically induced transparency [16]. The time scale here describes an incoherent flow of energy through the medium. The experiments of Ref. [13] however, did not discuss the contribution, to the multiple scattering dynamics, of the atomic alignment produced in atomic excitation with polarized light. In the studies reported here, we have determined the dynamics of the atomic alignment produced in an ultracold gas of ${ }^{85} \mathrm{Rb}$ under conditions similar to those reported in [13]. Measurements include observation of spectral 


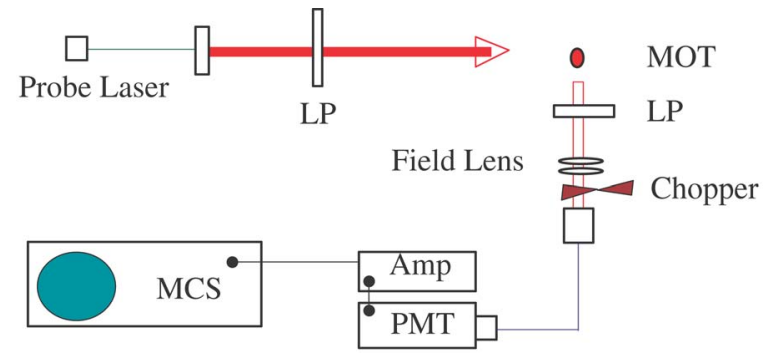

FIG. 1. (Color online) A schematic diagram of the experimental arrangement. Shown is a MOT, linear polarizers (LP), and a photomultiplier tube (PMT). Data are time-binned and stored in the multichannel scalar (MCS).

variations of the alignment in a range of several natural widths $(\gamma)$ around the atomic resonance, and of the time evolution and polarization of the multiply scattered light. The data are compared with Monte Carlo simulations of the processes, and found to be in good quantitative agreement. The most important result is that interferences among hyperfine scattering amplitudes strongly influence light propagation in the atomic gas, and may significantly impact efforts to obtain strong localization in an optically dense atomic sample.

As in Fig. 1, the experiment is performed on an ultracold gas of atomic ${ }^{85} \mathrm{Rb}$ prepared in a magneto-optical trap (MOT) operating on the $F=3 \rightarrow F^{\prime}=4$ hyperfine transition. The trap, which has been described elsewhere [17], produces a nearly Gaussian cloud of $\sim 10^{8}$ ultracold rubidium atoms at a temperature $\sim 100 \mu \mathrm{K}$. The peak density is $\sim 3$ $\times 10^{10} \mathrm{~cm}^{-3}$. The Gaussian radius of the sample is $r_{0}$ $\sim 1 \mathrm{~mm}$, determined by fluorescence imaging. Measurement of the spectral variation of the transmitted light gives a peak optical depth of $b_{0}=8(1)$. For a Gaussian atom distribution in the trap, the maximum weak-field optical depth is given by $b_{0}=\sqrt{2 \pi} n_{0} \sigma_{0} r_{0}$. Here $n_{0}$ is the peak trap density and $\sigma_{0}$ is the on-resonance cross section. The isolated-resonance scattering cross section $\sigma$ varies with probe frequency, $b=b_{0}[1$ $\left.+(2 \Delta / \gamma)^{2}\right]^{-1}$, where $\Delta=\omega_{L}-\omega_{0}$, and $\omega_{L}$ is the probe frequency, $\omega_{0}$ is the $F=3 \rightarrow F^{\prime}=4$ hyperfine transition frequency. A weak probe laser is tuned in a range of several $\gamma$ around this transition. The laser is a continuous wave diode laser having a bandwidth $\sim 1 \mathrm{MHz}$, and an average light intensity of $1 \mu \mathrm{W} / \mathrm{cm}^{2}$. To produce a nearly Gaussian beam profile, the laser output is passed through a single-mode optical fiber. The beam is then expanded and collimated to a $1 / e^{2}$ width $\sim 8 \mathrm{~mm}$. The probe laser intensity is modulated with an acousto-optic modulator (AOM), which generates nearly rectangular pulses having an on time of $2 \mu$ s and an off time of $2 \mathrm{~ms}$. The $2 \mu$ s excitation pulse is centered in a $90 \mu$ s window during which fluorescence signals are recorded. The MOT lasers are off during this period. For the remaining nearly $2 \mathrm{~ms}$, the MOT lasers are turned back on to reconstitute the atomic sample. Fluorescence from the MOT region present during this period is prevented from reaching the photomultiplier tube (PMT) by a synchronized mechanical chopper. The AOM-limited $20 \mathrm{~dB}$ response is $\sim 60 \mathrm{~ns}$. The probe laser is vertically polarized.

Scattered light signals are detected in a direction orthogo-

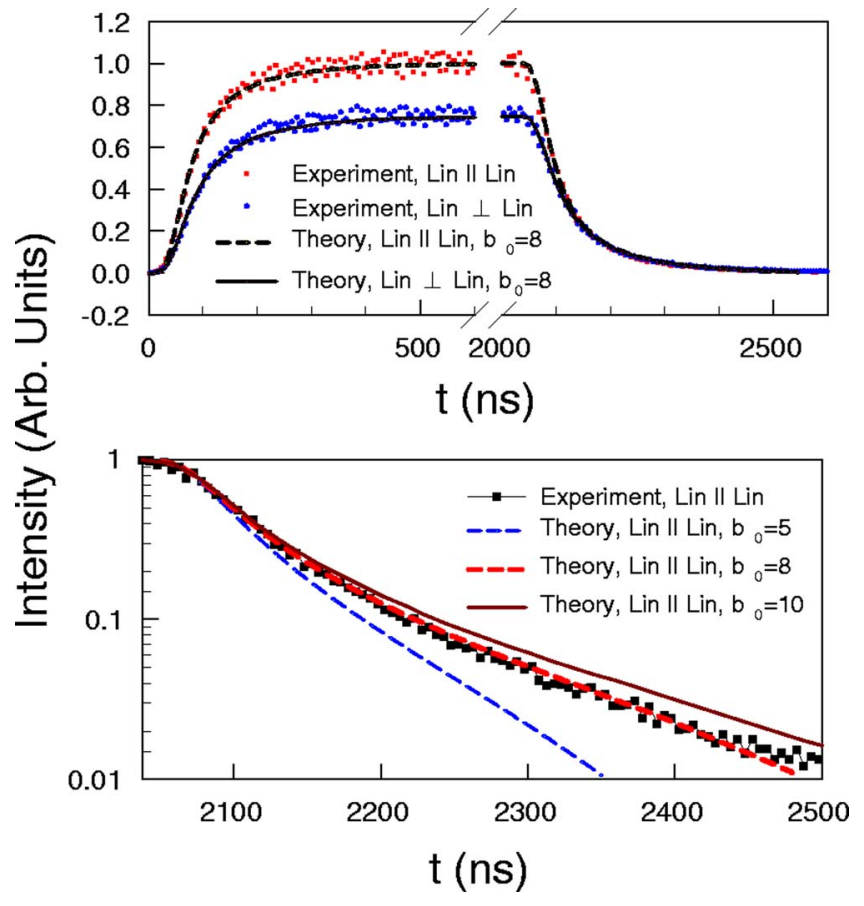

FIG. 2. (Color online) Measured time-dependent scattered light signal in orthogonal $P_{L}$ channels. Theoretical results are indicated by the curves, as labeled in the figure legend. The peak intensity corresponds to about $10^{4}$ counts.

nal to the probe laser propagation and polarization directions. The light is collected in a solid angle of about $0.35 \mathrm{mrad}$, and refocused to match the numerical aperture of a $400 \mu \mathrm{m}$ multimode fiber. A linear polarization analyzer is placed between the MOT and the field lens to collect signals in orthogonal linear polarization channels, labeled as parallel (II) and perpendicular $(\perp)$. The polarization response is calibrated against the known polarization direction of the probe laser; the measured $20 \%$ difference in sensitivity is used to correct the signals taken in the two channels. The fiber output is coupled through a $780 \mathrm{~nm}(5 \mathrm{~nm}$ spectral width) interference filter to a GaAs-cathode PMT. The PMT output is amplified and directed to a discriminator and multichannel scalar, which serves to sort and accumulate data into $5 \mathrm{~ns}$ bins. A precision pulse generator is used to control timing of the MOT and probe lasers and for multichannel scalar triggering.

The measured intensities in two orthogonal polarization channels for resonance excitation of the $F=3 \rightarrow F^{\prime}=4$ hyperfine transition are shown in Fig. 2. Note that the peak intensity in the lin $\|$ lin channel is very nearly $10^{4}$ counts, which corresponds to 10 experimental runs, each with a $120 \mathrm{~s}$ data accumulation period. The time response of the data acquisition system, including the AOM switching, is fast in comparison with the time evolution of the fluorescence signals. We point out that the transient buildup of several hundred ns is due to multiple scattering of probe radiation after it is switched on. The time scale for the process can be seen more clearly in the lower panel of Fig. 2. The first 50 ns of this curve is distorted by the electronic shutoff of the probe pulse. Beyond that, the decay curve is multiexponential, and varies 
from the natural single atom fluorescence from atoms located near the surface of the sample to longer-time-scale decay arising from atoms deeper within the sample. The solid curves in Fig. 2 represent Monte Carlo simulations of the scattering process. Other than the overall intensity scale, there are no adjustable parameters in the comparison, with the simulation input data consisting of the measured trap density profile and the AOM response. The agreement is excellent, showing that the physics of the process is well modeled.

The fluorescence time behavior given in Fig. 2 suggests for longer times an approximately exponential decay, with estimated time constant of 170(20) ns. It is generally expected that the longest time scale reflects the sample geometry, and is given by a single exponential, often termed the lowest-order Holstein mode [18]. In this regard, our results are in qualitatively good agreement with the those of [13] for our optical depth $b \sim 8(1)$. In [13], it is also shown that the longest decay time, in an elastic diffusion theory and for large optical depth, scales for a Gaussian atom distribution, as $\tau_{0}=0.057 \tau_{\text {nat }} b^{2}$, where $\tau_{\text {nat }}=27 \mathrm{~ns}$ is the natural decay time of the excited level. Although this result gives qualitatively good agreement with experiment, it seems to underestimate, at lower optical depths, the measured decay time, both in our results and in those of [13]. This difference may be due to departure of our atomic sample from an ideal Gaussian atom distribution, or to approximations made in the boundary conditions of the diffusion model [4].

From Fig. 2 it is also clear that the fluorescence signals are different for the lin $\|$ lin and lin $\perp$ lin polarization channels. This effect is quantified by defining a linear polarization degree as

$$
P_{L}=\frac{I_{\|}-I_{\perp}}{I_{\|}+I_{\perp}}=\frac{-15\left\langle A_{0}\right\rangle}{28-5\left\langle A_{0}\right\rangle} .
$$

In the formula, $I_{\|}$and $I_{\perp}$ represent the measured intensities in the lin $\|$ lin and lin $\perp$ lin channels. We emphasize that $P_{L}$ is related to the electronic alignment generated by excitation of an initially unpolarized atomic gas of ground-state atoms with linearly polarized light. Then only the average axially symmetric alignment component $\left\langle A_{0}\right\rangle$ is nonzero. The alignment is defined in terms of the upper state hyperfine angular momentum operators as the ensemble average $\left\langle A_{0}\right\rangle=\left\langle 3 \hat{F}_{z}^{\prime 2}\right.$ $\left.-\hat{\mathbf{F}}^{\prime 2}\right\rangle / F^{\prime}\left(F^{\prime}+1\right)$. In $P_{L}$ above, the expression in terms of $\left\langle A_{0}\right\rangle$ is correct for small detunings from the resonance line, where contributions from the $F=3 \rightarrow F^{\prime}=2,3 \rightarrow F=3$ transitions may be ignored. Finally, we point out that the above discussion ignores inelastic Raman transitions to the lower $F=2$ hyperfine level, which have a negligible effect on the reported data. The data in Fig. 2 give the time dependence of $P_{L}$ shown in Fig. 3 . There we see that $P_{L}$ enhances the differences in the two channels, showing the time-dependent maximum in $P_{L}$ soon after the exciting pulse is turned on. This is followed by the approach to a steady state $P_{L}$, which decays rapidly upon switching off the exciting laser pulse. The varied behavior can be understood by considering that when the exciting laser is first turned on, the prompt signal comes mainly from single scattering events. Then the peak
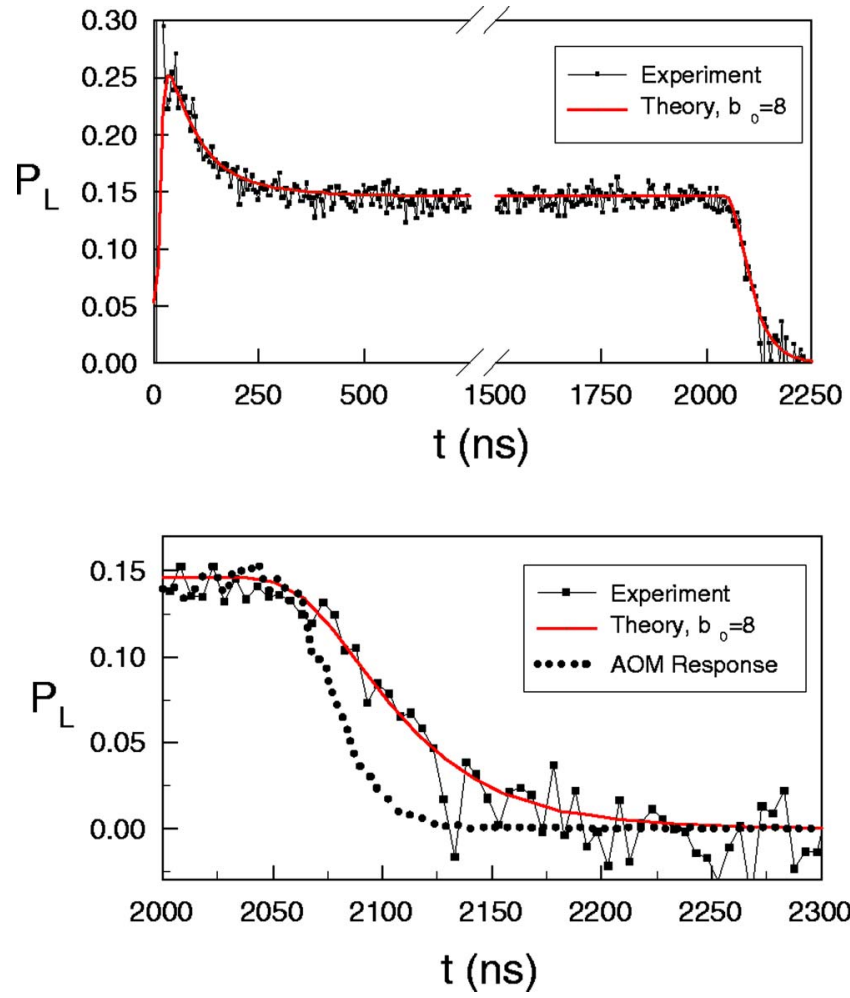

FIG. 3. (Color online) Measured time-dependent $P_{L}$, shown as solid points. Monte Carlo simulation results are shown as solid lines, while the limiting AOM response is shown as a dotted line.

value of $P_{L}$ should be close to the single scattering value of $P_{L}=0.268$, as is seen in Fig. 3. Second, even though the light scattering is nearly elastic, the polarization state of the scattered light will be randomized in multiple scattering by the presence of the multiplicity of available elastic Raman and Rayleigh radiative channels. Note that inelastic Raman transitions to the lower $F=2$ level are negligible in the spectral range of data reported here. Then we expect (and observe) that the steady state polarization, which has contributions from multiple order scattering events, is lower than the single scattering value, but is still nonzero. However, as seen in the lower panel of Fig. 3, once the excited laser is turned off, $P_{L}$ rapidly decays to a very small value. Note that the AOM-determined shutoff time for the excited light is not negligible on the scale of the $P_{L}$ decay. However, it is clear that, after a few atomic radiative lifetimes, $P_{L}$ has decayed to a small value. The decay is monotonic, and roughly exponential, with a decay constant on the order of two natural lifetimes. This is to be contrasted with the decay of the total excitation (see Fig. 2), where the population survives for much longer time scales. The alignment generated by optical excitation is then fragile in comparison with the population. Finally, theoretical results [19] for the decay rate of $P_{L}$ as a function of $b_{0}$ show that $P_{L}$ decays more slowly as the optical depth is reduced. This is physically plausible as, in a single scattering limit, $P_{L}$ would remain constant as the atomic population decayed.

As shown in Fig. 4, we have recorded data for a detuning range of $\pm 24 \mathrm{MHz}$ around the $F=3 \rightarrow F^{\prime}=4$ hyperfine transition. We see that, as the magnitude of the detuning is made 


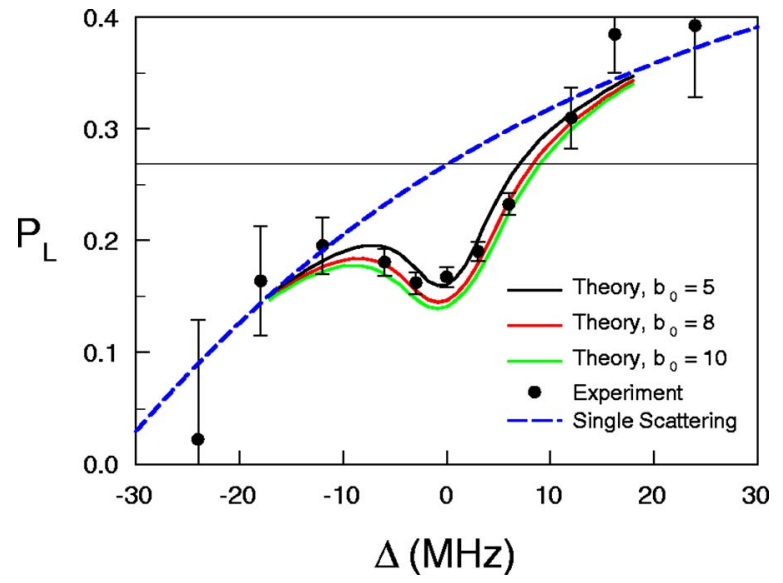

FIG. 4. (Color online) Detuning dependence of $P_{L}$. The dots represent experimental data points, while the chained curve is the expected variation for single atom scattering. The curves indicate variation for optical depths of $b_{0}=5, b_{0}=8$, and $b_{0}=10$.

larger, the measured steady-state values for $P_{L}$ approach the calculated frequency-dependent single scattering limit. This limit varies with detuning because of interference of the scattering amplitudes among the $F=3 \rightarrow F^{\prime}=2,3,4$ hyperfine transitions, and does not depend on the existence of multiple hyperfine levels in the $5 \mathrm{~s}{ }^{2} S_{1 / 2}$ lower energy level. In the absence of interference, $P_{L}$ is nearly constant, as indicated by the horizontal line in Fig. 4. The single scattering curve is expected, for as the laser is tuned away from resonance, the optical depth decreases, and the number of contributing scattering orders decreases. The solid curves in Fig. 4 represent theoretical results for different optical depths $b_{0}$. We see that the experimental data (with one sigma error bar) is bracketed by the $b_{0}=5$ and $b_{0}=8$ calculations, consistent with the experimental on-resonance optical depth of $b_{0}=8(1)$.

In conclusion, we have reported frequency, time, and polarization-dependent measurements of near-resonance fluorescence emitted, in a multiple scattering regime, from ultracold atomic ${ }^{85} \mathrm{Rb}$ atoms. The measurements are in excellent agreement with Monte Carlo simulations of the process. The results show that light scattered from the atomic ensemble maintains, for a time scale of several atomic lifetimes, a residual of the initial electronic alignment created by excitation with linearly polarized light. The steady state polarization varies strongly in the vicinity of atomic resonance, demonstrating that hyperfine interferences in the scattering amplitudes play a critical role in light transport in a dense atomic gas.

This work was supported by the National Science Foundation (Grant No. NSF-PHY-0355024), the Russian Federation for Basic Research (Grant No. RFBR-05-02-16172-a), and the North Atlantic Treaty Organization (Grant No. PSTCLG-978468).
[1] P. W. Anderson, Phys. Rev. 109, 1492 (1958).

[2] B. Damski, J. Zakrzewski, L. Santos, P. Zoller, and M. Lewenstein, Phys. Rev. Lett. 91, 080403 (2003).

[3] A. Krug, S. Wimberger, and A. Buchleitner, Eur. Phys. J. D 26, 21 (2003).

[4] P. Sheng, Introduction to Wave Scattering, Localization, and Mesoscopic Phenomena (Academic Press, San Diego, 1995).

[5] A. Lagendijk and B. A. van Tiggelen, Resonant Multiple Scattering of Light, Phys. Rep. 270, 143 (1996).

[6] D. S. Wiersma, P. Bartolini, Ad Lagendijk, and R. Righini, Nature (London) 390, 671 (1997).

[7] A. A. Chabanov, M. Stoytchev, and A. Z. Genack, Nature (London) 404, 850 (2000).

[8] M. D. Barrett, J. A. Sauer, and M. S. Chapman, Phys. Rev. Lett. 87, 010404 (2001).

[9] G. Labeyrie, F. deTomasi, J.-C. Bernard, C. A. Müller, C. Miniatura, and R. Kaiser, Phys. Rev. Lett. 83, 5266 (1999).

[10] G. Labeyrie, Ch. Miniature, and R. Kaiser, Phys. Rev. A 64, 033402 (2001); G. Labeyrie, C. Miniatura, C. A. Müller, O. Sigwarth, D. Delande, and R. Kaiser, Phys. Rev. Lett. 89,
163901 (2002).

[11] T. Chanèliere, D. Wilkowski, Y. Bidel, R. Kaiser, and C. Miniatura, Phys. Rev. E 70, 036602 (2004).

[12] D. V. Kupriyanov, I. M. Sokolov, and M. D. Havey, Opt. Commun. 243, 165 (2004).

[13] G. Labeyrie, E. Vaujour, C. A. Muller, D. Delande, C. Miniatura, D. Wilkowski, and R. Kaiser, Phys. Rev. Lett. 91, 223904 (2003).

[14] A. Fioretti, A. F. Molisch, J. H. Muller, P. Verkerk, and M. Allegrini, Opt. Commun. 149, 415 (1998).

[15] C. A. Müller and C. Miniatura, J. Phys. A 35, 10163 (2002).

[16] L. V. Hau, S. E. Harris, Z. Dutton, and C. H. Behroozi, Nature (London) 397, 594 (1999).

[17] D. V. Kupriyanov, I. M. Sokolov, P. Kulatunga, C. I. Sukenik, and M. D. Havey, Phys. Rev. A 67, 013814 (2003).

[18] T. Holstein, Phys. Rev. 72, 1212 (1947).

[19] D. V. Kupriyanov, I. M. Sokolov, C. I. Sukenik, and M. D. Havey, Laser Phys. Lett.(to be published), 10.1002/ lapl.200510059 (2005). 